\title{
Juego, desmadre y organización social: el caso de una barra de fútbol en México*
}

\author{
Ricardo Duarte Bajaña*
}

Recibido: 3 de octubre de 2019 • Aceptado: 3 de diciembre de 2019

\section{Resumen}

El objetivo de este trabajo es explicar las relaciones entre desmadre, juego y organización social en una agrupación de aficionados al fútbol, en contraste con la distancia que convencionalmente se ha señalado que tienen estas categorías. Los datos que sostienen este capítulo fueron obtenidos durante una investigación basada en un diseño etnográfico. Se estudió una agrupación denominada la Banda del Rojo, una de las barras de fútbol de la ciudad de Toluca, en el estado de México. El trabajo de campo fue realizado desde agosto del 2013 hasta abril de 2015. A partir de tres casos etnográficos se evidencia el desmadre-juego como parte de la organización social que los integrantes del grupo han construido en busca de una manera diferente de vivir a la impuesta en el hogar, en la escuela y en su contexto sociocultural. En este caso, el orden y el desorden dejan de ser polos opuestos y, en su lugar, formulan una manera de relacionarse que combina juego y jerarquía, turbulencia y organización social. En el análisis se confirma que el juego y el desmadre realizados por los

"Artículo de investigación. Proyecto financiado por una beca del Consejo Nacional de Ciencia y Tecnología de México y por una beca otorgada por la Universidad Iberoamericana de la Ciudad de México. "Game, 'desmadre' and social organization: the case of a soccer fans group in Mexico".

Citar como: Duarte, R. (2019). Juego, desmadre y organización social: el caso de una barra de fútbol en México. Revista de Investigación Cuerpo, Cultura y Movimiento, 10(1), 69-88. DoI: https://doi.org/10.15332/2422474x/5962

** Doctor en Antropología Social. Profesor, Universidad Autónoma del Estado de México. Toluca, México. Correo electrónico institucional: rduarteb085@profesor.uaemex.mx ridubaco@gmail.com. orcID: https://orcid.org/0000-0001-5436-7439 
integrantes de una agrupación mexicana de aficionados al fútbol se acercan a la anarquía, la libertad, la algarabía, la turbulencia y la diversión, y se articulan con una organización social rigurosa y jerárquica que ellos han construido.

Palabras clave: antropología del deporte, jóvenes, libertad, organización social, barras de fútbol. 


\section{Game, chaos and social organization. The case of a soccer supporters' group in Mexico}

Abstract

The purpose of this paper is to explain the relationships between "chaos" ("desmadre"), game and social organization in a soccer fans group, in contrast to the distance that conventionally has been indicated these categories have. The data that support this chapter were obtained during a research based on an ethnographic design. A group called la Banda del Rojo (The Red Gang), one of the soccer supporters' group of the city of Toluca, in the state of Mexico, was studied. The field work was carried out from August 2013 to April 2015. From three ethnographic cases, the chaos-game is observed as part of the social organization that the group members have built in search of living life in a different way from that imposed at home, at school and in their sociocultural context. It will be noted that, in this case, order and chaos cease to be opposites and instead formulate a way of relating that combines game and hierarchy, uproar and social organization. In this article it is argued that the game and the chaos ("desmadre") carried out by the members of a Mexican soccer supporters' group, are close to anarchy, freedom, racket, uproar and fun, and it articulates with a rigorous and hierarchical social organization that they have built.

Keywords: anthropology of sport, youth, freedom, hierarchy, social organization, soccer supporters' groups. 


\section{Introducción}

El desmadre ha sido objeto del interés investigativo de la antropología (Bartra, 2002; Magazine, 2008) y de la filosofía mexicana (Portilla, 1986). El desmadre ha estado asociado con la fiesta desenfrenada, el desparpajo, el consumo de alcohol en exceso y el albur ${ }^{1}$, entre otras características, y vincula el chiste y la ironía (Rosaldo, 1989), comportamientos que trasgreden la autoridad moral, el respeto y el control que la figura materna representa (Magazine, 2008). Sus raíces posiblemente se encuentren en la figura de "el pelado" (Franco, 1989; Bartra, 2002; Ramos, 2002), campesino urbano, despechado, resentido, quien frecuentemente es asociado a contextos de pobreza (Adler-Lomnitz, 1989; Lewis, 2013; 2014), que opera desde el “importamadrismo" (Bartra, 2002) y utiliza cotidianamente un lenguaje sin sentido para que solo "los de acá", es decir, las personas de su mismo grupo y clase social lo entiendan.

Para Bartra (2002), "el pelado" es una construcción que surge del vínculo entre el despotismo del Estado mexicano y la corrupción del pueblo. "El pelado" se constituye en una especie de comportamiento defensivo para vivir en un complejo y problemático contexto mexicano atravesado por la lucha de clases sociales evidenciada en las relaciones clientelares (Adler-Lomnitz, 1994; Adler-Lomnitz, Salazar, Adler, 2004). Para Bartra (2002), Cantinflas sería el "simulacro lastimero" de aquel contexto en donde viven dominantes y dominados, y en donde aquellos mexicanos doblegados por ciertas formas de poder utilizan el desmadre para relacionarse desde comportamientos transgresores.

El grupo de personas estudiadas durante esta investigación, quienes residen en la ciudad de Toluca, capital del estado de México, y quienes pertenecen a una agrupación organizada de aficionados al fútbol, realizan una serie de prácticas sociales que ellos mismos definen como "desmadrosas". Dentro de estas prácticas se encuentra la interpretación de canciones de manera desaforada y la ejecución del $\operatorname{slam}^{2}$ dentro de un ambiente festivo acompañado por el ritmo musical de la orquesta de la agrupación. Algunas de estas prácticas

1 Juego de palabras donde los participantes conversan utilizando ideas en doble sentido, frecuentemente con contenido sexual.

2 Es un baile asociado a ciertos géneros musicales (ska y rock en sus diversas vertientes), que consiste en saltar al ritmo de la música mientras se desplaza en el espacio empujando de manera descontrolada a los demás participantes. Sus protagonistas manifiestan que este baile representa diversión extrema y libertad. En el marco del argumento que se sostiene en este artículo, 
desmadrosas, realizadas por los integrantes de esta agrupación, pueden ser entendidas como juego por las siguientes razones: 1 ) se trata de acciones que tienen fines en sí mismas (Huizinga, 1998); 2) la emocionalidad es central durante su desarrollo; 3) durante su ejecución es muy importante el desprendimiento del esquema lógico de la vida cotidiana (Rivero, 2016; Caillois, 1986; Huizinga, 1998); 4) se caracterizan por la diversión, turbulencia, libre improvisación y despreocupada alegría (Caillois, 1986); 5) están acompañadas de un sentimiento de tensión y alegría (Huizinga, 1998).

El juego se ha entendido histórica y conceptualmente desde diferentes prespectivas (Enriz, 2011). Sin embargo, el juego y el "desmadre" usualmente han sido concebidos como la antípoda de la verticalidad, la rigidez, la jerarquía y la organización. Caillois (1986), por ejemplo, establece una distancia entre paidia y ludus, esto es, entre las manifestaciones espontaneas, libres, exuberantes, desordenadas y las formas de juego organizadas, disciplinadas, con virtudes civilizadoras. Desde esta perspectiva, el “desmadre” y el juego son la antítesis absoluta de la conciencia y de las formas de organización social. Esta interpretación conduce a extrapolar los comportamientos racionales, por un lado, y los irracionales y salvajes, por el otro.

El propósito de este artículo es explicar que, para la agrupación estudiada, esta división entre "desmadre-juego" y organización social no es tan clara y evidente, como lo han señalado algunos estudios clásicos acerca del juego (Duvignaud, 1980; Callois, 1986). Para los integrantes de esta barra de fútbol, aquellas actividades "desmadrosas", que tienen algunas características propias del juego, se enmarcan en una organización social que los integrantes de esta barra de fútbol han construido y la cual facilita el desarrollo de todas sus acciones colectivas (Duarte, 2019). Concretamente, lo que estos actores sociales denominan como "desmadre" puede ser analizado desde al menos tres interpretaciones: la primera es el "desmadre-juego" en sí mismo, aislado del contexto sociocultural y con unas dimensiones internas que lo constituyen. La segunda interpretación está asociada a la organización social del grupo que permite que ese "desmadre-juego" se lleve a cabo en un espacio y con unos participantes determinados. En este sentido, el "desmadre-juego"

los observadores externos ven en este baile caos, turbulencia y violencia. Sin embargo, sus actores señalan que no es violento y que las reglas son propias de la actividad. Es decir, la intensidad de los saltos y la fuerza de los empujones son dictadas por cada slam. 
ocurre gracias a un contexto sociocultural específico que conduce a lo que puede denominarse "organización del desmadre". Esta categoría conceptual implica que los comportamientos "desmadrosos" y lúdicos pueden tener fines y límites signados por la organización social del grupo. La tercera interpretación es la visión externa, esto es, la percepción de quienes no hacen parte de la agrupación y, por tanto, no están vinculados ni al “desmadre-juego" ni a la organización social del grupo. Ellos no están interesados en comprender las dinámicas de los participantes ni su organización social. Para la mayoría de estos últimos observadores el "desmadre-juego" es, justamente, un espectáculo salvaje, irracional, grotesco y violento que debe ser regulado y controlado por instancias gubernamentales. La atención de este artículo está dedicada, exclusivamente, a la segunda de estas interpretaciones.

Teniendo en cuenta esta segunda interpretación, resulta interesante la utilización de un concepto muy similar al de "organización del desmadre" en la investigación que Susana Rostas (2012) realiza con los concheros $^{3}$ en la Ciudad de México. Ella utiliza la idea de anarquía ordenada a partir de los planteamientos de Evans-Prichard (1940). Justamente, este último autor caracteriza el sistema político de los $\mathrm{Nuer}^{4}$, en Sudán, como una anarquía ordenada; si bien esta sociedad africana no tiene gobierno, ni organización política, ni liderazgo, su organización social se basa en su sistema de parentesco (Evans-Pritchard, 1940). Por su parte, Rostas (2012) señala que la anarquía ordenada de los concheros en México implica la conformación de grupos que no tienen una organización política o un liderazgo fijo y estable, sino que cambian y evolucionan constantemente.

Si bien la barra de fútbol investigada no tiene un gobierno o una organización política sistemática, sí mantiene un sistema estable de liderazgos. No obstante, lo que se quiere resaltar acá aludiendo al término anarquía ordenada (Evans-Prichard, 1940; Rostas, 2012) es la articulación entre la idea de anarquía y de organización que, aparentemente, poseen contenidos irreconciliables; de manera muy similar a como puede verse la "organización del desmadre”. El grupo de aficionados al fútbol de quienes se trata este texto

3 Grupos de personas que realizan una danza ritual en México que vincula tradiciones prehispánicas y cristianas. Los danzantes llevan el torso desnudo y utilizan tocados de plumas, ayoyotes en los tobillos que funcionan como sonajeros y la piel adornada con pintura de diversos colores.

4 Los Nuer son una sociedad africana ubicada principalmente en lo que hoy se conoce como Sudán del Sur. Su forma de vida se desarrolla en torno a la ganadería. 
realiza acciones que pueden ser entendidas como juego desde la conjugación entre organización y desmadre, esto es, no desde la anarquía total, pero tampoco desde una organización estructural absoluta. Vale la pena señalar que este argumento ya ha sido explorado en trabajos previos (Monsiváis, 1986; Magazine, 2004; Rivero, 2016). Específicamente, el juego y el desmadre realizado por los integrantes de esta agrupación se acercan a la anarquía, la libertad, la algarabía, la turbulencia y la diversión, aun cuando su organización social tenga componentes rigurosos y jerárquicos. Se trata entonces de una especie de orden desordenado.

\section{Metodología}

Los datos que sostienen este capítulo fueron obtenidos durante una investigación basada en un diseño etnográfico (McLeod y Thomson, 2009; Hernández, Fernández y Baptista, 2010) que permitió describir, analizar y comprender ideas, significados y prácticas de los integrantes de la Banda del Rojo, una de las barras de futbol de la ciudad de Toluca (Duarte, 2017) que apoya al equipo de fútbol denominado los Diablos Rojos del Club Deportivo Toluca. El trabajo de campo fue realizado desde agosto del 2013 hasta abril de 2015.

Esta investigación se sostiene en el método comparativo (RadcliffeBrown, 1975; Palerm, 1987; Sartori y Morlino, 1994), a partir del cual se establecieron diferencias y semejanzas acerca de la organización social y del sentido que tienen las relaciones sociales en dos agrupaciones de aficionados al fútbol localizadas en un mismo contexto: la ciudad de Toluca.

Los datos históricos de esta agrupación y de su contexto sociocultural fueron obtenidos con base en información testimonial de diferentes informantes. Los datos etnográficos se obtuvieron mediante observación participativa dentro de las actividades que se organizan al interior de la barra, así como a través de entrevistas no estructuradas y a profundidad aplicadas a algunos integrantes de la agrupación. Estos datos etnográficos fueron recabados en un diario de campo. El análisis de los datos se realizó siguiendo la "no directividad”, planteada por Rosana Guber (2008); es decir, se siguió las secuencias lógicas que los informantes señalaban para explicar su propia realidad. En este mismo sentido, se buscó el significado local de los conceptos construidos históricamente por la población estudiada. 
La información que acá se presenta no corresponde necesariamente a una traducción lingüística de lo que dicen y hacen los informantes, sino que, siguiendo a Bloch (1998), se trata de la expresión de unos postulados que orientan parte de sus vidas y que ellos valoran como fundamentales a partir de sus prácticas y diálogos, y que, a lo largo del trabajo de campo, el investigador aprendió a reconocer.

\section{Resultados}

\section{Organización social en la Banda del Rojo}

La agrupación de aficionados llamada la Banda del Rojo se conformó el 21 de febrero de 2004. Los creadores de este grupo intentaban oponerse a la manera autoritaria y vertical como se tomaban decisiones en la barra oficial denominada La Perra Brava. Esta última barra se conforma por personas adultas con fuertes relaciones con los directivos del Club Deportivo Toluca y con políticos mexicanos. Hoy en día, este tipo de relaciones prevalecen. Los jóvenes iniciadores de la Banda del Rojo también se oponían a este tipo de relaciones por considerar que conducían a "venderse" a estructuras de poder. Uno de los argumentos que permanentemente señalaban los informantes durante las entrevistas y conversaciones realizadas en el marco de esta investigación consistía en que los líderes de La Perra Brava actuaban solamente movidos por el beneficio económico que pudieran obtener. Se trata de un contexto problemático atravesado por relaciones clientelares (Duarte, 2017).

Mientras tanto, los jóvenes de la Banda del Rojo sostenían que, más allá del dinero, es la emoción la que debe guiar los actos dentro de la agrupación. Cabe precisar que algunos líderes e integrantes de esta barra se lucran con la reventa de boletos; sin embargo, estas prácticas son puntuales, la inmensa mayoría de sus miembros hacen parte de este grupo por razones diferentes al beneficio económico. En realidad, uno de los motivos fundamentales para que se vinculen y se mantengan en la Banda del Rojo es el "desmadre", que es visto como una salida para fugarse de las relaciones sociales centradas en las normas y la consecución de dinero para poder vivir. Se trata de una práctica social cercana a la propuesta por Bartra (2002), según la cual grupos sociales doblegados por determinadas formas de poder ven en el aquel una manera de relacionarse desde comportamientos transgresores. Estos jóvenes asocian este 
tipo de relaciones clientelares a la forma de interacción adulta, representada en los padres, jefes y profesores.

A partir de estos argumentos, decidieron conformar una nueva agrupación y empezaron a promover una actitud que ellos definían como "loca y rebelde" que contrastaba con los comportamientos más recatados de la agrupación hegemónica. Hoy en día, la Banda del Rojo cuenta con más de 1000 integrantes, en su mayoría menores de 25 años. La diferencia más destacada entre estas dos agrupaciones es la permisividad frente al desmadre. Mientras que en La Perra Brava se regula e incluso se prohíben los comportamientos desenfrenados, por ejemplo, el consumo de alcohol, el uso constante de groserías y las peleas, en la Banda del Rojo estos comportamientos son aceptados y valorados, siempre y cuando se cumplan las acciones programadas (asistir a reuniones, ir a los estadios en donde juega el equipo, participar en caravanas, firmas de autógrafos por parte de los jugadores, etc.), y no se pongan en riesgo los objetos valorados por el grupo (banderas, trapos $^{5}$, instrumentos, entre otros).

La Banda del Rojo se organiza en grupos denominados sectores o grupos de personas cuya residencia se ubica en barrios de la ciudad de Toluca o municipios aledaños. En términos concretos, la Banda del Rojo está conformada por la unión de múltiples sectores. El sector, como estrategia organizativa, fue desarrollándose paulatinamente, es decir, no surgió de una planeación racionalmente analizada y consensuada para después ponerla en ejecución y evaluación. En cambio, fue progresando con base en la necesidad que tenían los líderes de la Banda del Rojo de convocar adeptos que se convirtieran en parte de la agrupación.

Esta barra tiene tres líderes principales y cada sector tiene su líder particular. Los integrantes de cada sector eligen a su líder, así como los líderes de los sectores y los aficionados sostienen a los líderes de la agrupación. Esta elección y sostenimiento dependen de la capacidad que demuestren "moviendo" el sector y la barra. El concepto mover tiene que ver con la autoridad "desmadrosa" para convocar a sus integrantes a reuniones y actividades, con la facultad para promover el amor y la emoción por la agrupación, y con la capacidad de gestión administrativa y logística evidenciada en la consecución

5 Avisos de tela en donde se exhibe el nombre de la agrupación de aficionados y en donde se resaltan frases e imágenes de apoyo al equipo de fútbol que se sigue. En algunas ocasiones, se incluye la imagen de alguno de los jugadores importantes que ha tenido el equipo. 
de boletos, transporte y dinero en casos extremos, por ejemplo, para pagar fianzas para sacar de prisión a algunos de sus compañeros. Respecto a este último punto, cabe anotar que la policía detiene a algunos de estos jóvenes, comúnmente, por "echar desmadre" en el estadio.

Uno de los medios de comunicación que más frecuentemente se utiliza para "mover" u organizar las actividades en la Banda del Rojo es WhatsApp. Sin embargo, también tienen grupos de Facebook en el que suben fotografías después de cada partido en el que juega el equipo de fútbol de Toluca. Estos grupos son cerrados. Una parte importante de los datos etnográficos obtenidos a lo largo del trabajo de campo fueron conseguidos gracias a la vinculación del investigador a algunos grupos en estas redes virtuales.

Uno de estos "sectores" en donde se realizó el trabajo de campo durante esta investigación se ubica en el municipio de Otzolotepec, específicamente en la población de Villa Cuauhtémoc, ubicada a 35 kilómetros del estadio Nemesio Díez, de la ciudad de Toluca. Debido a que el lugar de residencia de la mayoría de sus integrantes es Villa Cuauhtémoc, los miembros de este "sector" decidieron denominarse la Banda del Rojo Villa o Los de Villa. Durante la investigación, esta pequeña agrupación se conformaba por 15 jóvenes. Sus edades oscilaban entre los 14 y 25 años. Este sector de la Banda del Rojo es relativamente joven, pues se constituyeron como tal en 2013. Mientras se realizó el trabajo de campo, su líder era Alan ${ }^{6}$. Llama la atención que ocho de sus integrantes se encontraban estudiando en universidades del estado de México. Dentro de las carreras que cursaban estaba Enfermería y Derecho. Incluso, algunos de ellos ejercían la profesión que estudiaban.

\section{Desmadre y juego}

En este marco de organización social, los integrantes de la Banda del Rojo valoran las acciones desmadrosas que son reprimidas en otros ámbitos de la vida social. Karla, una de las integrantes de Los de Villa, mencionó una idea que ya habían expresado otros jóvenes de la Banda del Rojo durante diferentes entrevistas y conversaciones, y que tenía que ver con las razones que les hace mantenerse dentro de esta agrupación: "En la barra puedo sacar lo que traigo

6 Con la intención de proteger su identidad, los nombres de los informantes fueron cambiados. 
de toda la semana“ (Karla, 19 años, comunicación personal, enero de 2015). Tratando de explicar esta justificación, Karla me dijo que algunos jóvenes "se quieren desahogar", buscan grupos adonde "nadie los juzgue" por alentar a un equipo, por cantar y bailar. Una vez inician su participación en la Banda del Rojo empiezan a sentir "gusto" por hacer cosas y tener sensaciones que en su vida escolar y familiar no experimentan, por ejemplo, cantar, gritar desaforadamente "hasta llegar a sentirse libres" (Karla, comunicación personal, enero de 2015), saltar, tomar licor, enfrentarse con grupos de aficionados que le van a otros equipos de fútbol o desafiar y huir de la policía.

El tipo de experiencias y sensaciones que pueden buscar los jóvenes que se vinculan a la Banda del Rojo pueden explicarse a partir de lo que Karla observó en una de sus amigas de la Banda del Rojo Villa:

Ese domingo jugaba el Toluca, mi amiga llegó al quiosco [lugar de encuentro], venía muy confundida, lloraba y no podía hablarme, yo no podía entender lo que me decía, estaba temblando. Yo le pregunté qué le había pasado, pensé que la habían robado. Ella me dijo que acababa de tener una gran discusión con sus papás porque ellos no querían dejarla ir ese día al estadio porque tendrían visitas y tenía que ayudar a limpiar la casa. Le dio mucho coraje y se escapó de su casa. Cuando se calmó me dijo que estaba harta de las reglas que le querían chingar en la escuela y en su casa. Ese día fue con nosotros al estadio y fue emocionante ver que gritó y saltó como loca hasta que volvimos. (Karla, comunicación personal, enero de 2015)

Karla recordaba, conmovida y al borde de las lágrimas, aquel incidente. Me dijo que, hasta ese momento, pensaba que nadie sentía lo que ella sintió cuando empezó a acompañar a la gente de la Banda del Rojo en el estadio. Me explicó que ella, en muchas oportunidades, tembló y se enfermó por no poder reunirse con su grupo para ir al estadio porque sus papás se lo prohibían.

Esta explicación permite comprender que la vinculación a una agrupación organizada de aficionados es una estrategia para realizar acciones atravesadas por la emoción que buscan distanciarse del esquema lógico del hogar y de la escuela en donde se imponen reglas inapelables.

A continuación, se exponen tres casos etnográficos en donde se evidencia el desmadre-juego dentro de la agrupación en un contexto de organización social que ellos han construido a lo largo del tiempo y de la experiencia buscando vivir la vida de manera diferente a la impuesta en el hogar y en la escuela. 
Vale la pena llamar la atención hacia la articulación y desplazamientos que el juego y el orden tienen en cada caso.

\section{Carnaval sobre ruedas}

Durante la semana previa a los partidos que el equipo de los Diablos Rojos juega en la ciudad de Toluca, los integrantes de cada sector, incluyendo a los de Villa, tienen un intenso cruce de información tanto presencialmente como a través de redes sociales. El objetivo de estas conversaciones consiste en organizar asuntos logísticos; uno de los más importantes consiste en reunir dinero - que todos los miembros aportan- para pagar un autobús que debe ser contratado para que los transporte hasta el estadio.

El día del encuentro deportivo, una vez surtidos los rigurosos trámites correspondientes y ya dentro del automotor, justamente cuando el sector ingresa a Toluca, es posible presenciar una especie de ritual que recurrentemente realizan los de Villa. Sin que mediara palabra, los muchachos dentro del autobús se desplazan de adelante hacia atrás y viceversa buscando trapos, extendiendo banderas e instalando tambores sobre los espaldares de los asientos. Mientras tanto, otros jóvenes se apresuran a abrir las dos escotillas que se encuentran en el techo del automotor. Una vez lograda esta tarea, tres personas salen por cada una de las escotillas, se sientan sobre el techo dejando sus piernas colgando hacia el interior del vehículo. Se puede ver a algunos muchachos transitando por el pasillo intentando encontrar un espacio para subir y sentarse en la parte exterior. Seguidamente, algunas personas le pasan banderas a quienes se encuentran sentados en la parte exterior. Al mismo tiempo, otros jóvenes abren las ventanas de los dos lados del vehículo y sacan más banderas y trapos por las ventanas. Los tambores empiezan a sonar desaforadamente acompañando los cánticos que practicaron durante la semana anterior y que incrementan su volumen mientras el autobús avanza por las calles de Toluca, guiado por el conductor que mira de soslayo por el retrovisor con una sonrisa cómplice. Al cuestionar a un joven, a quien le dicen Toques y quien se encontraba de pie sobre uno de los asientos acerca de la razón de aquel repentino aumento en el ánimo, explicó que lo hacían porque ya habían entrado a Toluca, y era necesario hacer notar a Villa. Efectivamente, la mayoría de los transeúntes volteaba a mirar aquel carnaval sobre ruedas. 
Sin embargo, a medida que aumentaba la euforia ya no era posible hablar con nadie. Todos cantaban y agitaban banderas y trapos con mucha fuerza. Los encargados de los tambores decidían cambiar de ritmo cada cierto tiempo, lo que generaba que todos cambiaran rápidamente de canción incrementando el desmadre. Se trata de una especie de juego que combina la mímesis y el vértigo. Es casi un trance colectivo que disminuye paulatinamente su potencia cuando llegan al lugar de destino, unas cuadras antes del estadio.

Durante el trabajo de campo, algunos integrantes de los de Villa señalaron en varias ocasiones que a ellos les gustaba formar parte de la Banda del Rojo porque dentro de la agrupación "nadie nos juzga". Interpretando sus comentarios recabados durante entrevistas no estructuradas, acciones como salir del autobús por las escotillas mientras cantan con mucha fuerza y agitan banderas también puede entenderse como una manifestación frente a las lógicas de la vida cotidiana y frente a una forma toluqueña recatada y conservadora de vivir la vida apuntalada en el cumplimiento de reglas y en la importancia que tiene el dinero.

Además, estos jóvenes critican ciertas prácticas propias de los adultos que están centradas en la posibilidad de hacer negocios y obtener beneficios económicos a través del futbol. Sin embargo, desconocen las necesidades y construcciones sociales que realizan los jóvenes aficionados que apoyan al equipo del Club Deportivo Toluca y para quienes las prácticas colectivas, emocionales y "desmadrosas" son fundamentales.

\section{Una nueva canción}

Domingo, una hora antes de ingresar al estadio Nemesio Diez de la ciudad de Toluca para presenciar un importante partido cuyo protagonista es el equipo de los Diablos Rojos se reúnen todos los sectores que conforman la Banda del Rojo en el parque Vicente Guerrero, ubicado a unas cuadras del estadio. Después de saludos efusivos entre amigos de diferentes sectores la orquesta de la barra, denominada Ridíkulos 26, empezó a tocar con mucha fuerza los tambores y las trompetas. Cuatro jóvenes caminaban rápidamente entre la multitud juvenil repartiendo pequeños papeles con letras verdes impresas en donde se leían cuatro estrofas: "Dale, dale, dale. Diablos Rojos / suden la camiseta / queremos otra vuelta dar. / Esta banda ya aguantó 10 años / los malos resultados / y acá estamos de fiesta. / Siempre alentamos / vayas donde vayas ahí estamos. 
/ ¡Oooh! Deportivo / eres lo más grande rojo querido” (extracto del diario de campo, 20 de julio de 2014). Se trataba del nuevo cántico de la agrupación que algunos de sus integrantes habían estado componiendo durante las semanas previas. Los minutos siguientes se dedicarían a un ensayo colectivo en el que participarían los aproximadamente quinientos jóvenes que allí se encontraban.

La orquesta repetía una y otra vez la misma melodía mientras que los asistentes miraban el pequeño papel y repetían las estrofas. Esta actividad era liderada por varios jóvenes de la agrupación quienes, ordenada y sistemáticamente, caminaban mirando a los presentes y cantando la canción y vocalizando más. Al mismo tiempo movían uno de sus brazos incitando a que se cantara con ánimo. Diez minutos después se matizó la actividad, cantando otros temas reconocidos dentro de la barra. Sin embargo, periódicamente se volvía a interpretar la nueva canción. Ya nadie leía el papel que contenía las estrofas y los jóvenes entonaban aquellas nuevas líneas con una emocionalidad creciente. Pasaron aproximadamente diez minutos más, sin dejar de tocar, y los miembros de la orquesta empezaron a caminar hacia la avenida Morelos, vialidad que conduce al estadio, en medio del camino que empezaron a despejar los participantes. El recorrido hacia el escenario deportivo se convirtió en una especie de carnaval atravesado por bombas de humo multicolor, banderas ondeantes, mientras la orquesta toca estridentemente y los integrantes de la agrupación cantan con el alma puesta en cada estrofa.

Una vez la orquesta arribó a la esquina de la avenida Morelos con la calle Aurelio Venegas, justo al pie del estadio, un tumulto de jóvenes la rodearon y empezaron a corear la canción que minutos antes habían aprendido. El volumen de la interpretación lindaba con el estruendo, con la turbulencia. Aquellos cantores saltaban al ritmo de la interpretación apoyando sus manos sobre los hombros de sus vecinos. Sus saltos eran enormes, cantaban con fuerza, casi con rabia, frunciendo todos los músculos de su cara y con los ojos cerrados. Daba la sensación de que la vida misma fluía en cada estrofa. Este trance colectivo disminuyó su intensidad cuando la orquesta empezó a dirigirse lentamente hacia la entrada del estadio para los controles y requisas de rigor.

A lo largo del aprendizaje de esta canción hasta su puesta en escena en la esquina del estadio, la organización fue disminuyendo su control y dio paso al desmadre, que se convirtió en una especie de juego en donde la conciencia ya no existía. Aquellos cantores saltadores solo existían en la canción, en el desmadre absoluto. 
Durante una entrevista realizada a Alan, manifestó que con este tipo de acciones los chavos que forman parte del sector que él lidera quieren liberarse de la presión que experimentan en su familia y en la escuela; contextos donde prima el cumplimiento de deberes y se inculcan unos modelos de comportamiento signados por la seriedad y el recato. Según él, la Banda del Rojo les da la posibilidad de librarse de estas responsabilidades. Sin embargo, Alan enfatiza que los integrantes de esta agrupación también tienen que cumplir con ciertas tareas dentro del sector. Uno de los problemas que enfrenta en su papel de líder consiste, justamente, en que algunas personas quieren echar desmadre todo el tiempo, hecho que se evidencia al llegar tarde a las reuniones, afirmar que asistirán al estadio y retractarse sin avisar, creer que formar parte del sector significa andar de fiesta y no participar en los ensayos, en la preparación de trapos y banderas, etc.

Frente a estas actitudes "desmadrosas" que dificultan las tareas del sector, Alan asume comportamientos mucho más serios. Incluso, ha llegado a reprender enérgica y públicamente a quienes no equilibran sus acciones entre el desmadre y su organización.

\section{Mimicry en el autobús}

Frecuentemente, cuando algunos jóvenes de la agrupación se reúnen, realizan juegos de simulación. Asumen el papel de cantantes, imitan artistas o a aficionados de otros sectores. Un ejemplo de estas prácticas ocurrió después de concluir un partido dentro de un autobús de regreso de uno de los estadios de fútbol de Ciudad de México. Sara, una de las integrantes de los de Villa, empezó a hacer comentarios con contenido sexual y todos dentro del vehículo reían estrepitosamente. La siguió otra joven que se encontraba sentada en la mitad del autobús; hacía referencia a que alguien le había "lastimado el clítoris”, todos reían nuevamente. Después de algunos comentarios de este tipo, Rogelio, el novio de Sara caminó desde la parte de atrás hasta adelante, adonde se encontraba su novia y empezaron a besarse apasionadamente mientras las personas cercanas se burlaban desaforadamente. Esta algarabía incrementaba la pasión de los besos de los novios.

Después, esta pareja caminó hasta la parte trasera del automotor, diciendo que iban a tener relaciones sexuales y todos continuaban abucheando a la pareja. En el último asiento el joven se sentó y la mujer también sobre él 
moviéndose seductoramente; simulaban tener una relación sexual, todos reían e intentaban observar cada detalle de aquel espectáculo.

Al cabo de un rato, la joven se puso de pie y caminó hacia adelante del bus, hasta donde se encontraban sus amigas; mientras caminaba simulaba secarse el sudor de la frente y decía que la actividad había estado agotadora pero que no había sido suficiente y que su novio debería mejorar su rendimiento. Todos volvían a reír, pero en esta ocasión se burlaban del joven que se había quedado en la parte de atrás del autobús haciendo gestos con los que simulaba estar exhausto.

En ese momento, Juan, uno de los jóvenes, empezó a gritar que Sara aguantaba y que en realidad se había cansado muy rápido. Luego le preguntó a Sara que si Paola, una de las aficionadas que se encontraba sentada en la parte de la mitad del automotor, tendría que esperar mucho. Todos se reían estrepitosamente, aplaudían y empezaron a gritar “¡Beso!, ¡beso!”. En aquel punto Sara se levantó de su asiento y regresó caminando seductoramente por el pasillo central hacia donde se encontraba Paola. Esta última se levantó en medio de la algarabía de los presentes. Cuando se acercaron lo suficiente, se dieron un apasionado beso en los labios. El éxtasis colectivo era incontrolable, los observadores reían, aplaudían y saltaban. Cuando Sara y Paola terminaron de besarse, Sara le gritó a Juan que quién seguía porque todavía no estaba cansada. Todos continuaban riendo desaforadamente. Sara regresó a su asiento, junto a sus amigas y lentamente los ánimos se calmaron en medio de chistes y conversaciones respecto a lo que acababa de ocurrir.

A lo largo de toda esta representación, cuyas reglas se iban construyendo colectivamente, lo que ocurría en la calle no existía para estos jóvenes. Toda la atención estaba puesta en aquel espectáculo que despertaba la euforia de los presentes. Otro aspecto significativo es el realismo y la vitalidad de los actores; en realidad, parecía como si estuvieran representando una comedia de la que todos hacían parte.

Durante la semana siguiente, en una entrevista a profundidad que se le realizó a Paola, se le indagó acerca del apasionado beso que había protagonizado con Sara en el autobús. Según ella, esos comportamientos son vistos de manera negativa solamente por los padres de familia o por los profesores. Sus respuestas coincidieron con algunos comentarios que habían realizado durante la investigación otros jóvenes residentes en Villa Cuauhtémoc. Ellos consideran a su municipio como un "pueblo rascucho", es decir, un pueblo 
feo y atrasado. Ven en la Banda del Rojo una posibilidad para vivir de una manera diferente, con más libertad, sin tanta presión ejercida por la familia y por creencias católicas.

\section{Discusión}

En el campo de las investigaciones de las barras de fútbol, algunos investigadores han evidenciado la preocupación por definir como emoción las prácticas de estos grupos (Archietti, 2003). El problema, según ellos, consiste en que este camino conduce a tratar como puramente salvajes e irracionales a sus participantes, lo que construye e intensifica una escisión entre razón y emoción. Este tipo de distanciamientos se puede observar, por ejemplo, en el trabajo de Duvignaud (1980): "La conmemoración es a la fiesta lo que la regla es al juego: una tentativa de establecimiento social para absorber, digerir o apropiarse debilitándolo, aquello que lo uno y lo otro tienen de inaceptable para el orden establecido" (Duvignaud, 1980, pp. 139-140).

Sin embargo, agrupaciones de aficionados al fútbol como la Banda del Rojo intentan mostrar permanentemente que hay otra forma de ver las cosas. Sus prácticas, si bien son profundamente lúdicas, también tienen un componente racional y estrictamente organizativo. El problema, entonces, consiste en pretender distanciar la razón de la emoción, asignando a estos conceptos valoraciones morales divergentes.

Teniendo en cuenta las enseñanzas de la Banda del Rojo y siguiendo los planteamientos de Bartra (2002), se puede aseverar que el desmadre - $y$ también el juego, en este caso- es una especie de pequeña revolución privada que, al fin de cuentas, no amenaza el orden impuesto por el Estado. Sin embargo, la agrupación de aficionados estudiada va más allá, y muestra lo que acá se ha denominado la "organización del desmadre", donde el orden y el desorden dejan de ser polos opuestos y, en su lugar, formulan una manera de relacionarse que combina juego y jerarquía, turbulencia y organización social. Para la gente de la Banda del Rojo esta aparente contradicción no existe. Para ellos, organización y desmadre pueden ir juntos, lo que lleva su práctica a un nivel diferente al que plantean los análisis tradicionales.

Si bien la estructura social, en principio, acapara toda la atención de los integrantes de esta agrupación, su poder se va difuminando — sin 
desaparecer- para dar paso a la turbulencia, el desmadre y el descontrol propios del juego. La enseñanza de los jóvenes de la Banda del Rojo entra en sintonía con lo planteado por Ivana Rivero y que consiste en la necesidad de "enseñar a disfrutar de la incertidumbre, de crear un orden desordenado a los ojos de la rigidez adulta" (Rivero, 2016, p. 61).

\section{Conclusiones}

El "desmadre" es una manera de relación social (Bartra, 2002) que permite que los jóvenes se vinculen y se mantengan en la Banda del Rojo como una salida para fugarse de relaciones sociales centradas en las normas, la consecución de dinero para poder vivir y el clientelismo que ellos asocian con el mundo adulto representado en los padres, jefes y profesores.

Sin embargo, esta agrupación se basa en una organización social con fuertes redes de comunicación jerárquica entre diferentes líderes y aficionados. Esta organización permite el diseño, asignación y cumplimiento de tareas que se evidencian masivamente cuando juega el equipo de fútbol de Toluca.

Lo que hacen permanentemente los integrantes de la Banda del Rojo es articular lo racionalmente organizativo con el juego y el desmadre. Concretamente, sus acciones como barra son una forma de organizar el desmadre, o de "anarquía ordenada", si se trata de utilizar los términos empleados por Evans-Prichard (1940) y Susana Rostas (2012). Esto es, con un rumbo en mente deciden perderse en la algarabía, la turbulencia y el colorido. Teniendo un fin, un propósito, sus acciones lúdicas se rebelan a los objetivos eminentemente productivos de la vida, al control de los padres y profesores. Se trata de una forma de vivir dentro del ocio, que articula permanentemente lo que en apariencia se escinde en la literatura del juego: la paidía y el ludus (Caillois, 1986).

Si se los analiza desde afuera y en sí mismos, esto es, desconectados del contexto, los juegos que realizan los integrantes de la Banda del Rojo son "desmadrosos", emocionales y turbulentos; son una fiesta, en términos de Duvignaud (1980). Sin embargo, lo que proponen los integrantes de esta agrupación es la necesidad de comprender la fortaleza de un contexto organizativo muy particular que enmarca y permite que estos juegos desmadrosos se desarrollen. 


\section{Referencias}

Adler-Lomnitz, L. (1989). Cómo sobreviven los marginados. México: Siglo XXI.

Adler-Lomnitz, L. (1994). Redes sociales, cultura y poder: ensayos de antropología latinoamericana. México: Flacso.

Adler-Lomnitz, L., Salazar, R. y Adler, I. (2004). Simbolismo y ritual en la política mexicana. México: Editorial Siglo XXI Editores.

Archietti, E. (2003). Masculinidades. Fútbol, tango y polo en la Argentina. Buenos Aires: Antropofagia.

Bartra, R. (2002). La jaula de la melancolía. México: Conaculta.

Bloch, M. (1998). How we think they think. Anthropological approaches to cognition, memory and literacy. Nueva York: Routledge.

Caillois, R. (1986). Los juegos y los hombres: La máscara y el vértigo. México: Fondo de Cultura Económica.

Duarte, R. (2019). Organización social, desmadre y libertad: críticas al neoliberalismo y propuestas juveniles en una barra de futbol mexicana. En J. Pedraza, K. Brito, E. Cortés, Juventudes latinoamericanas: perspectivas desde la interdisciplinariedad (pp. 171-192). Manizales: Cinde.

Duarte, R. (2017). "Mover el sector" y “desmadre organizado": clientelismo y corporativismo contemporáneo visto desde las agrupaciones de aficionados al fútbol en Toluca, Estado de México [tesis no publicada de doctorado]. Universidad Iberoamericana, Ciudad de México.

Duvignaud, J. (1980). El juego del juego. México: Fondo de Cultura Económica.

Enriz, N. (2011). Antropología y juego: apuntes para la reflexión. Cuadernos de Antropología Social, (34), 93-114. DoI: https://doi.org/10.34096/cas.i34.1382

Evans-Pritchard, E. (1940). The Nuer: a description of the modes of livelihood and political institutions of a Nilotic people. Oxford: Oxford University Press.

Franco, J. (1989). Plotting women: gender and representation in Mexico. Nueva York: Columbia University Press.

Guber, R. (2008). El salvaje metropolitano. Reconstrucción del conocimiento social en el trabajo de campo. Barcelona: Paidós.

Hernández, R., Fernández, C. y Baptista, P. (2010). Metodología de la investigación. México: McGraw-Hill.

Huizinga, J. (1998). Homo Ludens. Madrid: Alianza.

Lewis, O. (2013). Antropología de la pobreza. México: Fondo de Cultura Económica. 
Lewis, O. (2014). Los hijos de Sánchez. México: Fondo de Cultura Económica.

Magazine, R. (2004). ¡Es puro desmadre! Desorden y violencia entre jóvenes de un club de futbol en la ciudad de México. JOVENes, Revista de Estudios sobre Juventud, 8(21), 40-53.

Magazine, R. (2008). Azul y oro como mi corazón: masculinidad, juventud y poder en una porra de los Pumas de la UNAM. México: Universidad Iberoamericana.

McLeod, J. y Thomson, R. (2009). Researching social change. Nueva York: SAGE Publications.

Monsiváis, C. (1986). iiiGoool!!! Somos el desmadre. Cuadernos Politicos, (47), 57-73. Recuperado de http://www.cuadernospoliticos.unam.mx/cuadernos/ contenido/CP.47/CP47.6.CarlosMonsivais.pdf

Portilla, J. (1986). Fenomenología del relajo. México: Fondo de Cultura Económica. (Original publicado en 1966).

Radcliffe-Brown, A. R. (1975). El método de la antropología social. Madrid: Anagrama.

Ramos, S. (2002). El perfil del hombre y la cultura en México. México: Espasa.

Rivero, I. (2016). El juego desde los jugadores. Huellas en Huizinga y Caillois. Enrahonar. Quaderns de Filosofia, 56, 49-63. DoI: https://doi.org/10.5565/ rev/enrahonar.663

Rosaldo, R. (1989). Culture and Truth: The Remaking of Social Analysis. Boston: Beacon Press.

Rostas, S. (2012). Carrying the world: The concheros dance in México. Denver: University Press of Colorado. 